\title{
El método de trabajo con los sueños según los testimonios de pase de Escuelas lacanianas afiliadas a la AMP y la EPFCL
}

\author{
Methodological Aspects of Dream Intervention Modes Referred to in Pass \\ Testimonies from Lacanian Schools Affiliated with the WAP and the SPFLF
}

\author{
Ma. Celeste Labaronnie ${ }^{1}$ \\ Dttps://orcid.org/0000-0003-2923-8218 \\ Maximiliano Azcona² \\ https://orcid.org/0000-0003-3024-8560 \\ 'Laboratorio de Historia de la Psicología, Facultad de Psicología, \\ Universidad Nacional de La Plata, Argentina \\ ${ }^{2}$ CONICET - Centro de Investigaciones en Filosofía, Facultad de Humanidades \\ y Ciencias de la Educación, Universidad Nacional de La Plata, Argentina
}

\begin{abstract}
Resumen. Objetivo. En este artículo se presenta un estudio de los modos de intervención frente a los sueños referidos en testimonios de pase de escuelas lacanianas afiliadas a la Asociación Mundial de Psicoanálisis y la Escuela de Psicoanálisis de los Foros del Campo Lacaniano. Método. Se constituyó una muestra intencional de 80 testimonios, recabados de las publicaciones de cada escuela. A partir de ahí, se analizaron cualitativamente los 14 ejemplos encontrados en los que se explicitaba el modo en el que el analista intervino sobre el relato de un sueño. Resultados. El resultado es el hallazgo de variadas intervenciones que fueron agrupadas en cuatro ejes: (a) la interrogación o invitación a asociar; (b) la interpretación; (c) el corte; (d) otras variantes del acto analítico. Se concluye que el trabajo con los sueños delineado por Freud sigue en pie, pero también se observan otros modos de intervención que apuntan a un más allá del sentido: a la letra y al corte como estructura fundamental de la interpretación.
\end{abstract}

Palabras clave. Sueño, interpretación, psicoanálisis, testimonio.

Abstract. Objective. This paper presents a study of dream intervention modes referred to in pass testimonies from Lacanian Schools affiliated with the World Association of Psychoanalysis and the School of Psychoanalysis of the Forums of the Lacanian Fields. Method. The intentional sample consists of 80 testimonies, obtained from the publications of each school. From that point, we analyzed qualitatively 14 examples, in which the intervention of the analyst over a dream story was explicit. Results. We could find varied interventions and gather them into four main ideas: (a) interrogation or invitation to associate; (b) interpretation; (c) cut; (d) other analytic act variations. As a conclusion, the Freudian approach with dreams is still in effect, but we also observe some others ways to intervene that aim beyond sense: to letter and to cut, as the foundations of psychoanalytic interpretation.

Keywords. Dream, Interpretation, Pychoanalysis, Testimony.

${ }^{1} \mathrm{Ma}$. Celeste Labaronnie. Instituto de Investigaciones en Psicología (IniPsi), Facultad de Psicología, Universidad Nacional de La Plata, Argentina. Dirección postal: A. E-mail: celelab@gmail.com

${ }^{2}$ Maximiliano Azcona. Instituto de Investigaciones en Psicología (IniPsi), Facultad de Psicología, Universidad Nacional de La Plata, Argentina. E-mail: azconamaxi@hotmail.como.com.

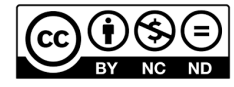

Esta obra está bajo una licencia de Creative Commons Reconocimiento-NoComercial-SinObraDerivada 4.0 Internacional. 


\section{Introducción}

En este artículo se presenta un estudio de los modos de intervención frente a los sueños referidos en testimonios de pase de algunas escuelas lacanianas nucleadas por la Asociación Mundial de Psicoanálisis (AMP), la Escuela de Psicoanálisis de los Foros del Campo Lacaniano (EPFCL) y la Escuela Freudiana de Buenos Aires (EFBA). El propósito de esta investigación es situar el método con que se trabajan actualmente los sueños en el psicoanálisis lacaniano. Con la expresión "trabajo con los sueños", se hace referencia a cualquier tipo de intervención del analista sobre el relato de un material onírico -sea que luego tenga efecto interpretativo o no-, y abarca tanto el pedido de asociaciones como la interpretación o el acto.

Podría decirse que, a partir de la práctica del pase, los sueños se han presentado a la comunidad analítica como material que "habla por sí mismo", que "dice" sin necesidad de ser interpretado, pues su virtud es la de sintetizar un caso entero en unas pocas líneas de relato onírico. Sin embargo, la pregunta sobre cómo se trabajan los sueños en el psicoanálisis lacaniano sigue en pie, pues habitualmente se intuye que las directivas freudianas no alcanzan para explicar los pasos de la cura lacaniana y que los sueños no se interpretan ya del mismo modo en que lo hacía Freud. Tampoco podría afirmarse que los sueños, como parecen sugerir a veces los testimonios, ya no se trabajan. Por lo tanto, en esta investigación la propuesta es rastrear las intervenciones del analista que sí figuran en los testimonios, para poder iluminar esos modos en que el sueño es intervenido, interpretado, puntuado, etc.

\section{El pase como dispositivo de investigación}

El dispositivo del pase fue propuesto por Lacan (1967/2012) como modalidad de organización para las escuelas de psicoanálisis que habían decidido regirse por su enseñanza, y también, como dispositivo para investigar el objeto y finalidad del análisis. Lacan esperaba que, a través de quienes se ofrecieran a testimoniar, el pase posibilitara el estudio de "los problemas cruciales en los puntos vivos en que se encuentran para el análisis, especialmente en tanto ellos mismos están en la tarea, o al menos en la brecha, de resolverlos" (Lacan, 1967/2012, p. 262). La implementación del pase debía producir un saber acerca de cómo resuelve cada cual el final de su análisis, qué efectos obtiene y qué restos incurables extrae.

Este dispositivo, tal como Lacan lo propuso, permite que cualquier analista que considere su análisis terminado, o se encuentre en un momento de atravesamiento, que ha sido llamado "momento de pase" (Lacan, 1973; Miller, 1973, 2007), pueda solicitar narrar su experiencia en una escuela de psicoanálisis. Se le asignan dos "pasadores" - analizantes que no han terminado sus curas, pero transitan un análisis avanzado-, que serán quienes escucharán el testimonio del candidato pasante y lo relatarán luego a un jurado integrado por miembros de la escuela. Esto implica que el testimonio no es narrado en forma directa, sino a través de los pasadores. Finalmente, el jurado puede otorgar, o no, la nominación de AE (Analista de Escuela). Si ese fuera el caso, en la actualidad se espera que, durante los años subsiguientes, el AE testimonie de manera directa sobre su análisis en diversos eventos o escritos, con lo cual se persigue el objetivo de profundizar el conocimiento de la comunidad analítica sobre los efectos del psicoanálisis. Esta práctica de los testimonios directos y públicos no estaba contemplada en la propuesta de Lacan y no se inició sino hasta hace algunas décadas. 
Actualmente, es muy habitual que esos testimonios se publiquen en diversos medios: revistas, libros, memorias de congresos o jornadas, entre otros. Este abundante material clínico, publicado por los mismos AE y relatado en primera persona, es útil para estudios como este, donde se utilizan únicamente testimonios que han sido publicados de forma abierta para todo público. El hecho de que se trate de publicaciones abiertas permite que todo investigador pueda utilizarlos sin necesidad de pedir consentimiento, pues se trata de un material disponible, entregado a la comunidad psicoanalítica en el mismo formato que cualquier texto teórico.

\section{El trabajo con los sueños desde una perspectiva lacaniana}

Como lo hemos señalado previamente (Labaronnie, 2015) en su artículo Retorno a la interpretación de los sueños, López (2009) distingue dos tipos de práctica lacaniana, que le parecen desprendidos de dos concepciones levemente diferentes de la noción de "letra". Para el autor, ambas orientaciones coinciden en que se trata de una noción forjada para nominar aquello del lenguaje que podría estar más cercano a su materialidad. Es decir que ambas consideran que esta noción es una formalización posible de aquello que hace límite y también empalma lo simbólico con lo real. No obstante, se diferencian por el lugar que confieren a los efectos de sentido.

Una de esas orientaciones, basada en la noción de letra como soporte material del lenguaje, habilitador del encuentro con nuevos sentidos (Lacan, 1957/2003), perfila el trabajo con los sueños hacia una técnica del significante, "como medio para producir el chisporroteo del sentido insospechado" (López, 2009, p. 133). La otra, en cambio, se basa en una concepción de la letra en tanto "marca", rasgo que inscribe el "fuera de sentido" (Lacan, 1971/2012), con el cual privilegia la práctica del corte en el abordaje del material onírico, para posibilitar el encuentro en acto del sujeto con el agujero que hay en su parloteo, y vaciar sus identificaciones imaginarias.

López no considera que ambas orientaciones sean inconciliables; antes bien, encuentra a la segunda concepción de letra, ya insinuada en los primeros trabajos de Lacan y, por lo tanto, no en ruptura, sino en continuidad con su primera propuesta. En especial, puntualiza el hecho de que la interpretación, para Lacan, ya desde 1957 "se opone a toda operación de decodificación de un sentido previo" (López, 2009, p. 134).

Lacan (1973-1974) sostenía que, si la cuestión gira en torno al sentido, desemboca siempre en un sentido sexual, como lo mostró Freud. Brousse (1997) fue una de las primeras en retomar este tema a partir de los sueños comentados en testimonios de pase. Los hallazgos producto de este dispositivo la llevaron a afirmar que "entre Freud y Lacan el lugar del analista dentro del tratamiento ha cambiado" (p. 28). Afirma que, puesto que el analista ya no opera a partir de la significación fálica, el despliegue de la misma queda a cargo del analizante.

Por su parte, Basz ha afirmado que "hay un saber hacer con los sueños que es distinto a interpretarlos. (...) Se trata fundamentalmente de situar en acto lo real como causa y la consecuente captación de un estallido inédito del estatuto del Otro" (2012, p. 101). A partir de Lacan, lo real ubicado como causa concierne al goce, al objeto y a la letra, pero no a la significación; lo cual conduce a un modo diferente de recibir los sentidos de la asociación libre en análisis.

Algunos analistas (Brousse, 1997; Cottet, 2000; Klotz, 2000; Basz, 2012) han considerado que esta orientación hacia la reducción de los sentidos es la causante de que los sueños relatados en los testimonios sean tan escuetos y valgan más por unas pocas ideas que el soñante asocia que por lo que el analista haya interpretado. 


\section{Los sueños en los testimonios de pase}

Desde que el dispositivo del pase comenzó a implementarse en las escuelas, la importancia de los sueños ha sido ampliamente destacada. Se llegó a hablar de una omnipresencia de sueños en los testimonios (Brousse, 1997) y en general las escuelas han coincidido en que los pasantes los utilizan para dar cuenta de los virajes más importantes de la cura (Brousse, 1997; Nepomiachi, 1999; Cottet, 2000; Farías, 2011;Thamer, 2018).

La cuestión ha sido estudiada desde diversos aspectos: el valor de ciertos sueños como criterio de finalización del análisis (Cottet, 2000), su carácter interpretativo (Brousse, 1997), las consecuencias conclusivas que los soñantes extraen de ellos (Thamer, 2018; Yacoi, 2002, 2012), su articulación con el fantasma (Nepomiachi, 1999; Rojas, 2017; Soler, 1988), con la satisfacción propia del fin de análisis (Mazzuca, 2011) y el tipo de despertar que acarrean (Nemirovsky, 2004; Naparstek, 2005; Koretzky, 2012).

Por el contrario, las intervenciones del analista sobre dichos sueños no han sido objeto de estudio sistemático. Inclusive podría decirse que los testimonios de pase han coincidido tanto en el relato de sueños breves, auto-interpretados y conclusivos, que el movimiento lacaniano ha corrido varias veces el peligro de olvidar que sin el método freudiano no hay análisis posible. Sin embargo, la hipótesis de esta investigación apunta a que un retorno a las intervenciones del analista que figuran en los testimonios permitirá afirmar que el pedido de asociaciones y la interrogación de los elementos del sueño siguen vigentes.

\section{Método}

\section{Uso de los testimonios en el presente estudio}

Se constituyó una muestra intencional de 80 testimonios, a partir de las publicaciones de tres escuelas principales: Asociación Mundial de Psicoanálisis (AMP), Escuela de Psicoanálisis de los Foros del Campo Lacaniano (EPFCL) y Escuela Freudiana de Buenos Aires (EFBA). La intención fue recabar tantos testimonios como fuera posible, sin operar en este primer momento ninguna discriminación. Se incluyeron publicaciones tanto en español como en francés.

En un segundo momento, se aplicó un procedimiento de selección basado en el siguiente criterio: testimonios en los que se explicita el modo en el que el analista interviene sobre el relato de un sueño. Con este paso, la muestra se redujo a solo 14 ejemplos, provenientes de 12 testimonios, tres de ellos pertenecen a la EPFCL y nueve a la AMP, los cuales se consignan en la Tabla 1.

\section{Método de análisis}

Sobre esta muestra se realizó un análisis cualitativo, en el cual se siguieron coordenadas teóricas psicoanalíticas, que permitieron identificar tipos de intervenciones y categorizarlas. En el procedimiento de análisis de los datos se adoptaron algunos de los principios generales de la teoría fundamentada, como el análisis comparativo constante (Strauss \& Corbin, 2016). Se construyó una codificación inicial mediante el análisis y comparación de los testimonios de pase, que luego fue cotejada con categorías teóricas y metodológicas de la teoría psicoanalítica, lo cual da lugar al surgimiento de las categorías e hipótesis interpretativas que aquí se comunican.

Especialmente, se consideró el efecto de las intervenciones según las palabras de los implicados, de acuerdo con las afirmaciones que constan en estos documentos de dominio público. 
Tabla 1

Testimonios de pase utilizados en esta investigación

\begin{tabular}{ll}
\hline Escuela & Texto de referencia \\
\hline $\begin{array}{l}\text { Escuela de Psicoanálisis de los Foros del Campo } \\
\text { Lacaniano (EPFCL) }\end{array}$ & Dahan P. (2010) \\
& Mazzuca, M. (2011) \\
& Vidal, C. (2015) \\
Asociación Mundial de Psicoanálisis (AMP) & Fajnwaks, F. (2016) \\
& Ferreira da Silva, R. (2013) \\
& Gasbarro, C. (2014) \\
& Horne, B. (2012) \\
& Lutterbach, A. L. (2009) \\
& Palomera, V. (2006) \\
& Rabinovich, D. (2015) \\
& Tarrab, M. (2006) \\
& Vinciguera, R.-P. (2006) \\
\hline
\end{tabular}

\section{Resultados}

El análisis cualitativo realizado sobre los testimonios ha permitido clasificar las intervenciones del analista en cuatro categorías: la interrogación o invitación a asociar; la interpretación; el corte; otras variantes del acto analítico. Estos ejes no son mutuamente excluyentes, y tampoco pueden pretenderse exhaustivos, pero permiten organizar los hallazgos y circunscribir la discusión conceptual. Su armado se desprende de decisiones tomadas por los investigadores con el propósito de organizar el material al seguir lineamientos clásicos del psicoanálisis, tales como el método freudiano de interrogación de los elementos discretos del relato onírico; la interpretación, tanto en su vertiente freudiana como la versión más "a la letra" que se desprende de la práctica de Lacan; el corte, como modalidad de interpretación preferida por este último; y, finalmente, lo que Lacan (1958/2005) defendió en textos como La dirección de la cura con "otras variantes del acto analítico" que denotan la libertad a nivel técnico/táctico.

\section{La interrogación o invitación a asociar}

Esta es la técnica fundada por Freud (1900/2000), desarrollada en el capítulo dos de La interpretación de los sueños. La extracción de los ejemplos que aquí se trabajan permite afirmar que el pedido de asociaciones y la interrogación de los elementos del sueño siguen vigentes, y que tal vez sean la base para muchos de los efectos que luego son apreciados con más claridad a partir de otro tipo de intervenciones.

El método descrito por Freud (1900/2000, pp. 122-125) consiste, en primer lugar, en no tomar al sueño entero como objeto de análisis, sino a cada uno de sus fragmentos por separado. También debe enseñarse al paciente a tomar nota de los pensamientos que acuden a él cuando se lo interroga respecto de cada fragmento y a comunicar lo que se le ocurre, sin sofocar aquellas ideas que le parezcan sin importancia o disparatadas. 
Para instrumentar este método, Freud concebía diferentes procedimientos técnicos: "proceder cronológicamente, y hacer que el soñante produzca sus ocurrencias sobre los elementos del sueño en la secuencia en que estos se presentaron en el relato del sueño" (1923/2000, p. 111); o bien "iniciar el trabajo de interpretación por un elemento destacado del sueño" (p. 111), que puede ser el más nítido, el más extraño o, por ejemplo, un dicho allí incluido. En tercer lugar, también es aceptable prescindir del contenido manifiesto y preguntar, en cambio, por los acontecimientos de la víspera, e iniciar el trabajo por allí. Por último, "cuando el soñante ya está familiarizado con la técnica de la interpretación" (p. 111) es posible dejar el trabajo a su cargo y confiar en el criterio con que va escogiendo las ocurrencias acerca del sueño con las cuales comenzar y proseguir el análisis.

En la muestra de 14 ejemplos se encontraron dos que pertenecen a esta modalidad de intervención. Uno de ellos proviene del testimonio de una pasante mujer (EPFCL), de sobrenombre "Cuca". Cerca del final de su análisis, ella relata el siguiente sueño: "Estoy sentada en una cama rodeada de cucarachas, quiero bajarme, pero no puedo porque si me bajo pisaría las cucarachas y si las piso hacen «CRAC». "¿Y que es crac?», pregunta la analista. "Un ruido». Fin de la sesión" (Vidal, 2015).

Este ejemplo sería también abordable en el eje (c), ya que combina la interrogación con un corte de sesión. No obstante, interesa detenerse en los señalamientos de otro analista acerca de este proceder interrogativo:

Si el analista hubiese comprendido demasiado rápido llenando de sentido el significante "cucaracha", este comprender no habría sido sino un "saber ingenuo", que habría encubierto el trazado de lo real impuesto al goce. (...) Hubiese sido el caso de un analista que, por ejemplo, hubiese interpretado que lo que ese significante crac quería decir era la representación del aplastamiento de su existencia por la preeminencia de esa frase fantasmática "pobre cuca". (...) El analista se detiene en un significante: crac. (Rojas, 2017, pp. 69-70)

Según se relata en el testimonio, la frase "pobre Cuca" había marcado toda su historia y había sido largamente comentada en el análisis. Lo que Rojas (2017) señala es que, en ese momento avanzado de la cura, la asociación del significante "crac" con la novela familiar no hubiese agregado ningún efecto novedoso, mientras que su extracción produce, en cambio, la revelación de una fijación libidinal en este significante onomatopéyico y todo su campo asociativo.

De la misma manera, en muchos testimonios se dice al pasar que en algún momento se tuvo un sueño y que "al asociar en sesión" se produjo cierta iluminación, una frase quedó resonando, o se impuso cierta idea o significante; pero, en general, no se menciona si el analista impulsó ese trabajo o cómo lo hizo; probablemente, porque los testimonios se centran en los últimos tramos de la cura, donde ya el analizante sabe trabajar de acuerdo con la cuarta de las modalidades propuestas por Freud.

Reparemos ahora en otro testimonio (EPFCL), de un analizante varón. El párrafo comienza con el relato de un sueño y continúa con una breve mención del proceder del analista y del efecto que produce el método de la interrogación:

Logro que me inviten y me hagan partícipe de una salida de mujeres. Vamos todas a cenar. Soy una mujer más entre las mujeres, lo cual me produce expectativas y una gran curiosidad por aquello de lo que hablan.

Al relatar este sueño en sesión [y como respuesta a los interrogantes del analista] constato que se produce un efecto de pérdida de lo que denominaría 
el "goce de la curiosidad". Dicho de otro modo: no hay grandes secretos, las mujeres no hablan de nada en particular, simplemente hablan (...). (Mazzuca, 2011, p. 44)

El interés de este ejemplo reside en que se menciona cuál fue la labor del analista en esa oportunidad y, además, no se la vincula con los efectos de verdad que se producen en el análisis -también sumamente importantes-, sino con algo menos frecuente: el agotamiento de la búsqueda. Se señala entonces cómo la interrogación puede muy bien conducir al agujero en el sentido: se interroga insistentemente acerca de qué espera el analizante encontrar en una cena de mujeres, hasta llevarlo a concluir que allí no habita ningún secreto respecto al goce. Algo similar se ha comentado en muchos testimonios, donde de un modo u otro, el analizante constata la inexistencia del "otro" -ya sea que se lo haya encarnado en la mujer, el saber totalizado o en cualquier otro imposible-.

\section{La interpretación}

Entre los testimonios estudiados se encontró uno (AMP) en el que se hace referencia a un tipo de interpretación netamente freudiana:

Soñé que me miraba al espejo. Veía mi cara tal como la de mi padre justo antes de afeitarse. ¡Un horror! ¡Así no se podía ir a ningún lado! El analista dijo: "Se quedó encerrada en las barbas de su padre". Ese sueño y su interpretación dejaron una huella. (Rabinovich, 2015, p. 89)

Esta interpretación, como podemos ver, es breve y tiene una estructura cercana al chiste (Freud, 1905/2000), ya que produce la sorpresa y el alivio propios de la agudeza. Podría decirse que es una interpretación freudiana clásica, pues a partir del contenido manifiesto, extrae una formulación lingüística que ha sido escogida por el sueño entre otras posibles, más abstractas y menos plásticas- por adecuarse mejor a los requisitos de figurabilidad (Freud, 1900/2000). La participación de un hábito idiomático es sumamente frecuente en estos procesos de cifrado y descifrado (p. 348). Si bien este tipo de interpretaciones son muy referidas y estudiadas en el ámbito lacaniano, en la muestra no se encuentran otras interpretaciones que puedan considerarse equivalentes; probablemente, porque en los testimonios suelen mencionarse los sueños que producen por sí mismos cierto efecto de interpretación.

Tanto para Freud (1912/2001) como para Faig (2012), el proceso de formulación de una interpretación pertenece al inconsciente. En su libro sobre el chiste, Freud desarrolla algunas ideas sobre el modo en que este es producido, que bien podrían valer para la interpretación. Resalta, por ejemplo, el carácter de emergencia involuntaria, de ocurrencia que prosigue a un estado de ausencia, de cese de la tensión intelectual. En ese estado, el chiste "brota de golpe, las más de las veces junto ya con su vestidura" (Freud, 1905/2000, p. 160). En cambio, cuando una alusión chistosa es producto de una intención consciente, formada bajo el control del "yo", no produce el mismo efecto. El chiste -al igual que la interpretación-, "aparece sin que yo sea capaz de perseguir en mi pensar esos estadios preparatorios" (Freud, 1905/2000, p. 161). De allí el efecto de sorpresa que tienen muchas interpretaciones, tanto para analista como para el analizante.

Continuando con los testimonios, un pasante (AMP) relata que con su primer analista ciertos sueños no fueron trabajados, ya que este propuso dar por terminado el análisis. Tiempo después, en un segundo tratamiento, esa secuencia de sueños es alojada y lo que recibe una nueva interpretación es el proceder del analista anterior: 
Le cuento al nuevo analista las coordenadas de ese final y tres sueños que había tenido los días anteriores a la última sesión. En el primero de esos sueños entraba a una habitación y me veía a mi mismo muerto. En el segundo sueño, mi padre me decía, mostrándome unos restos humanos descuartizados, que yo debía hacerme cargo de eso. En el tercero de los sueños yo estaba en mi consultorio, muy satisfecho, rodeado de objetos que representaban mis ideales. Con sorpresa veía en el diván un niño, un huérfano, al que abrazaba. (...) Esa secuencia, que no se dejó olvidar, fue por fin escuchada y mereció una primera interpretación: aquel analista cerró la puerta del análisis. Con esa interpretación se reabría esa puerta y yo entraba como un huérfano en la transferencia. (Tarrab, 2006, párr. 11)

Como se ve, lo que recibe una interpretación en este caso no es ninguno de los sueños relatados, sino el proceder del primer analista. Pero a la vez, la transferencia se constituye, al interpretarla, como una invitación a proseguir el trabajo. Aquí, la interpretación no es pospuesta hasta la instalación de la transferencia, sino que la comienza. Podría decirse que, indirectamente, se señala que esos sueños contienen información relevante.

Se transcriben a continuación otros sueños interpretados que se han encontrado en los testimonios, ordenados según una progresión que evidentemente apuntan a revelar cierta dimensión del goce. Luego, se introducen algunas reservas respecto a este tipo de interpretaciones, especialmente cuando apuntan a la pulsión y aplastan, de esa manera, el espacio del deseo. El primero de ellos (AMP), por ser enigmático, deja suficiente lugar para el movimiento deseante:

(...) estamos ambos mirando desde una ventana lo que sucede abajo: una pequeña niña patinando, con vestido de bailarina, en medio de una avenida repleta de autos. Me inquieto: ¡la van a atropellar! Pero enseguida la avenida deshace su recta y se convierte en un semicírculo hacia arriba, como una pista de skate, con el alivio consiguiente: ella está practicando. No hay peligro alguno (Gasbarro, 2014, p. 98).

Tras asociar el skate y los patines con un triciclo que tenía de pequeña, la soñante se pregunta: "Triciclo-patines, ¿qué era eso? El analista dice, como quien profiere una fórmula muy conocida; “¡Pues claro! ¡Es tu pasión por el deslizamiento!". Salí desconcertada. Jamás había sido tal pasión un tema del análisis. Pero luego comenzaron a caer algunas fichas" $^{\prime \prime}$ (p. 98-99).

Por lo que se desarrolla a continuación en el testimonio, es de suponer que la intervención toca tanto al placer del "deslizamiento" en el modo de hablar de esta paciente, como al deslizamiento en tanto actividad física. Esta interpretación la lleva finalmente a pensar que la traslación de un sentido a otro estaba impidiendo finalizar la cura. De alguna manera, allí algo del goce es tocado y modificado en su funcionamiento, lo que produce efectos duraderos.

En el siguiente testimonio (AMP) se comenta una intervención que invita al analizante a interrogar el valor libidinal de un elemento del sueño, producido por el mecanismo de la condensación:

${ }^{3 " C}$ Caer la ficha" es una expresión que en Argentina se usa para significar "darse cuenta" de algo, reparar en algo que no se había advertido.

Actualidades en Psicología, 34(129), 2020, 91-107 
El sueño mostraba: yo jugando a lanzar un disco blanco a un amigo que me lo devolvía y agregué: "Hay algo que sé, estoy en Bahía". Las asociaciones tomaron caminos diversos. (...) Al terminar la sesión, el analista dijo: "El disco blanco es una condensación". Sorpresa y, en un primer momento, desilusión. Tantas asociaciones interesantes y el analista dice algo tan banal. No obstante, trabajador decidido, al reflexionar, me doy cuenta del valor condensado de la propia interpretación. (Horne, 2012, p. 36)

En otro pasaje del testimonio, este pasante explica cómo entiende esa interpretación:

Al señalar la condensación el analista orienta el discurso desde el punto de vista económico y en la vertiente del objeto (...). Ese disco blanco, que era un frisbee con el que jugaba en la playa con un amigo, fue la primera versión del objeto a, forma de llamar el goce fijo del síntoma que en el sueño muestra el inicio de su puesta en movimiento que se reveló al final del análisis. (Horne, 2012, p.39)

Podría decirse que la pregunta que subyace a estas dos interpretaciones no es ¿qué representa este elemento del sueño?, sino ¿qué valor libidinal condensa? Su virtud reside en que, a la vez que llama la atención sobre el mismo, deja indeterminados los sentidos e invita a proseguir el trabajo. De esta manera, un sueño, que fue presentado tempranamente en la cura, es retomado cuando esta estaba llegando a su fin y permite nombrar algo del objeto en juego en el fantasma.

Lo mismo sucede con otra pasante, que transitó por varias curas, y relató el siguiente sueño a quien sería su último analista:

Hubo un primer sueño en el que quedaba como resto une poudre blanche. Un polvo blanco. En francés resuena la cocaína, en castellano lo sexual. Si bien ambas resonancias podían tener una relación conmigo, ninguna me cerraba. [El analista] aceptó sin insistir en ninguna de las dos alternativas nombradas y descartadas. Tranquilamente dijo: "Al final sabremos qué es la poudre blanche". Fue justamente la poudre blanche que apareció la anteúltima vez que lo vi. (Rabinovich, 2015, p. 93)

Lo que esta pasante señala es que ese analista captó que el significante que quedaba suelto condensaba un valor libidinal especial, aunque todavía no supieran de qué se trataba -al igual que ocurre en lo relatado por Horne-.

Más tarde, cuando la cura llegaba a su fin, una serie de sueños y la emergencia de un recuerdo infantil olvidado retoman aquel significante y lo ubican en el centro del síntoma. El polvo blanco era, en efecto, la sal con que la madre de la analizante mataba las babosas que devoraban las plantas del jardín. Allí resuena el nombre de la paciente -Débora- y su modo fantasmático de relación con el otro -el "pegoteo"- (Rabinovich, 2015). Puede verse que, si bien los sentidos no faltan, el énfasis está puesto en el valor libidinal de ciertos significantes y en el peso de la letra.

Por último, otro testimonio (AMP) da cuenta de un modo más directo de interpretación:

Luego de una proliferación de sueños de angustia llenos de detalles y de sentidos, surge un sueño simple -es una escena: un perro defecando un patê es mirado por un joven. Están en el sueño las diversas versiones del objeto a: objeto anal, objeto mirada, el falo y el objeto de la fobia infantil.

Actualidades en Psicología, 34(129), 2020, 91-107 
La interpretación sin sentido del analista: "ese patê es usted" (patê: significa paté, pero aquí es también una condensación de "para tener ${ }^{4 "}$ ). (Lutterbach, 2009, p. 22)

Cabe aclarar que si bien la paciente nombra esto como "interpretación desprovista de sentido", no consideramos que el sentido esté ausente en la frase del analista. Sin embargo, allí la interpretación no apunta tanto a los sentidos del significante "patê", como a subrayar el elemento de goce que el sueño revela.

Ahora bien, ¿qué valor tienen este tipo de interpretaciones para la cura? Soler (1988) ha señalado que la consideración del modo en que la pulsión se inserta en el sueño resulta "esencial para determinar la respuesta que conviene dar al sueño en la dialéctica de la cura, aquello a lo que, después de todo, el analista se ve confrontado cada día" (p. 75). Considera que el sueño, que habitualmente trabaja para plantear las preguntas del sujeto, a veces puede incluir una respuesta, al señalar el objeto causa de deseo y de goce para ese soñante. Lo que el analista hace con el tipo de interpretaciones mostradas anteriormente es señalar esa respuesta subjetiva, con el propósito de destacarla, ponerla de relieve para que pueda ser leída por el analizante.

Sin embargo, creemos conveniente aclarar que dentro del movimiento lacaniano, también se ha indicado que el abuso de este tipo de interpretaciones en la cura produce resistencia, debido a los efectos sugestivos que puede inducir:

Es inevitable en el análisis la revelación de algunos términos del vocabulario del goce (...). A estos significantes que inexorablemente se repiten (...) el analista los puede revelar; también los puede hacer equivocar en la interpretación (...); pero no insistirá sobre ellos en su decir sin que el analizante comience a presentarle su resistencia a la sugestión. (Lombardi, 1998, p. 33)

Lo que Lombardi señala como problemático es la insistencia en este tipo de interpretaciones, pero no su uso, que le parece en cierta medida necesario. También, propone que el analista puede, mediante el equívoco, enlazar esos términos que poseen valor libidinal con otros sentidos posibles. Un ejemplo de este proceder puede verse en el citado testimonio de Débora Rabinovich, donde se comenta que el analista, una vez despejada la relación del polvo blanco con el recuerdo infantil, introduce un equívoco sobre el significante "sal", retomándolo como imperativo del verbo salir: "¡Sal!" (p. 96). Así, se pasa de una ligazón mortífera de ese significante -veneno para matar babosas-, a un sentido vivificante, escuchado por la analizante como una invitación a salir de la novela familiar y del análisis mismo, para embarcarse en una nueva etapa de su vida.

La reiteración en los testimonios de este tipo de interpretaciones ha sido señalada por Brousse (1997) "de las pocas interpretaciones que quedan del analista en los testimonios, la mayoría procede bajo la misma lógica, es decir, bajo la lógica del equívoco que separa al sujeto de sus identificaciones idealizantes" (p. 25).

El corte

En este texto, se separa a la interpretación del corte, aunque por sus efectos deberían encontrarse en un mismo eje. Es así que las ideas que se desarrollan en este apartado, se encuentran en íntima relación con las discutidas en el anterior y son su continuación. A su vez, el lector puede haber notado que algunos de los ejemplos mencionados hasta

${ }^{4}$ La autora se refiere a las resonancias de la expresión en el idioma portugués, su lengua materna. 
ahora incluían al corte de sesión como modalidad táctica de intervención. Es el caso del sueño de las cucarachas comentado por Vidal (2015), donde la analista introduce una pregunta, escucha la respuesta y corta la sesión. Es también el caso del sueño relatado por Lutterbach (2009), del cual se ha omitido la continuación, donde se especifica que, tras proferir la interpretación, el analista corta la sesión (p. 22). También, se puede observar que los relatos de Horne (2012) y Gasbarro (2014) relacionan directamente la interpretación con el final de la sesión, aunque no mencionen explícitamente el corte.

Como dijimos, el corte no puede separarse de la noción de interpretación; debe pensárselo más bien como una de sus modalidades. "El corte es sin duda el modo más eficaz de la interpretación analítica", sostenía Lacan (1958-1959/2015, p. 537). Sin embargo, no toda interpretación asume manifiestamente la forma del corte. Muchas de ellas apuntan a desarmar significaciones que insisten, o bien operan sobre el significante, y si bien justamente por eso producen un efecto de corte, no consisten ellas mismas en introducir una pausa temporal en determinado momento del discurso, como sí observa en los ejemplos de este eje.

Naparstek (2001) sostiene que para Lacan el trabajo asociativo debía producirse entre encuentro y encuentro, ya que el circuito asociativo tiene tendencia a cerrarse sobre sí mismo -tanto por la elaboración secundaria que opera al despertar, como por la compulsión del yo a la síntesis, que interviene durante el trabajo analítico-.

Se ve que así se arma un trayecto, que va de la preparación del sueño al soñar y a la posterior interpretación, que gira en redondo pudiéndose hacer interminable. Esto último es lo que lleva a Lacan a poner énfasis en alguna estrategia que no deje al sujeto dando vueltas infinitamente. La misma está centrada en la escansión, el corte, lo discontinuo. (Naparstek, 2001, p. 147)

A su vez, este autor va a proponer que la escansión, como estrategia de intervención, tiene al menos dos objetivos. Por un lado, causar el trabajo nuevamente: causar el esfuerzo del analizante a seguir soñando y a seguir asociando. En este sentido, el analista operaría como causa de deseo, inclusive como resto diurno que pone a trabajar. Una precisión interesante que hace al respecto es que, para operar de esta manera, la intervención del analista debería quedar como "intramitada". Se refiere a que no podría ser una intervención que consista en una explicación o una respuesta a la demanda. De algún modo, la intervención debe incomodar al "yo".

El segundo objetivo del corte apunta efectivamente a lo que se ha extraído como despertar. En este casoya no se trata de relanzarcadavez, sino de dirigirse a ponerun fin, a detener el circuito. Allíel corte intenta despertar al sujeto para que pueda hacer algo diferente a seguir eternamente elaborando el resto. (Naparstek, 2001, p.148)

Entonces, este segundo propósito de la escansión es instar a "hacer algo", expresión en la que reconocemos el "saber-hacer-ahi" (savoir y faire) proclamado por Lacan (19751976/2009) como producto exigible de un análisis. Tal como lo explica este autor, aprender a hacer algo con los restos es diferente a seguir intentando elaborarlos.

A los ejemplos de uso del corte citados en los ejes anteriores, agreguemos ahora otros dos:

Después de varios años de análisis durante los cuales me parecía que nada se había movido, que nada significativo había cambiado en mí, tuve un sueño. La interrupción de la sesión sobre una palabra de las asociaciones del sueño produjo 
un efecto que calificaré de fulgurante. Esta palabra podía resumir un pequeño recuerdo de infancia que había sido interrogado a lo largo de mi análisis, pero en el momento no había hecho el lazo. Lo que hubo allí de fulgurante, es que el corte de la sesión había producido un efecto de choque y que a este significante del sueño inmediatamente asocié otro significante (...) Las sesiones que siguieron me permitieron asociar sobre una parte de mi historia que me parecía no me concernía. (Dahan, 2010, p. 28)

Como vemos, esta pasante (EPFCL) habla de "efecto fulgurante" y "efecto de choque" para calificar lo producido por el corte de sesión sobre un elemento de las asociaciones. Según comenta, el trabajo que se produce a partir de allí no difiere del trabajo asociativo habitual.

Lo que parecen lograr este tipo de intervenciones es hacer escuchar algo que formaba parte del discurso, pero cuya importancia no se advertía. Esto se ve destacado aún más en el ejemplo siguiente (AMP), donde una analizante de Lacan destaca un corte realizado, no durante el relato de las asociaciones, sino del sueño mismo: "'Estaba ${ }^{5}$ mi padre que...' El analista la interrumpió secamente; se escuchó ${ }^{6}$ a si misma al bajar la escalera haber dicho 'Yahvé mi padre'; ¿todo padre no es acaso Dios?" (Vinciguerra, 2006, p. 1).

Lo abrupto de este corte ilumina, según la pasante, la "figura de un padre con un goce sin límites" (párr. 7), que subyacía al amor del padre muerto y eternizado que la atormentaba desde hacía varias décadas. Lo relaciona con el segundo tiempo de la fantasía "Pegan a un niño" (Freud, 1919/1999). Como se observa, el corte produce un efecto de sorpresa; luego, es el analizante quien llena ese espacio vacío con nuevas asociaciones. Sin embargo, lo que queda del corte es cierta reducción.

\section{Otras variantes del acto analítico}

Finalmente, hay un grupo de ejemplos hallados en los testimonios que, si bien revisten valor interpretativo y por ello han sido recordados y relatados, se presentan como casos paradigmáticos de la "libertad táctica" propuesta por Lacan (1958/2005).

Agrupamos estos ejemplos bajo el título de "Variantes del acto analítico" porque se destacan por mostrar la acción del analista frente a ciertos sueños relatados, y constituyen maneras novedosas de recepcionar el material y darle un lugar en la cura.

El acto, definido por Lacan (1969/2012, p. 395) como "un decir a partir del cual el sujeto cambia", admite la extensión del término "decir" a toda acción que no pueda considerarse automática, sino fruto de una elección inconsciente, con consecuencias imposibles de prever. La interpretación, entonces, es una de las formas del acto, pero no todo acto se reduce a una interpretación. Así, la noción de "acto analítico" permite iluminar la acción del analista cuando, sin ser interpretativa, produce efectos en la cura.

Un pasante (AMP) recuerda:

Quería ser un buen analizante y esperaba entonces del analista una aprobación en relación a los sueños y a las asociaciones que producía en sesión. Él se encargó, a través de sus ruidos, tanto más fuertes en la medida en que yo buscaba actuar mi performance, de hacer deconsistir este circuito y hacer resonar el vacío que habitaba a menudo mi palabra (Fajnwaks, 2016, p. 78).

${ }^{5}$ En francés /l y avait (estaba) es homofónico de Yahvé.

${ }^{6}$ La pasante se refiere a sí misma en tercera persona, lo cual es habitual en algunos testimonios.

Actualidades en Psicología, 34(129), 2020, 91-107 
Vemos que el analista utiliza en este caso ruidos para incomodar aquello que en el analizante pujaba por producir un buen desempeño. Puede ser pensado como el "desdén" que recomendaba Lacan (1958/2005) para la hazaña obsesiva: el desinterés mostrado hacia cierto material, muy elaborado y racionalizado por el "yo", intenta vaciar ese espacio para dejar lugar al surgimiento de algo con valor más enigmático, propiamente inconsciente.

Se puede pasar a otro ejemplo de acto analítico en relación con los sueños. Una pasante mujer relata que su tratamiento atravesó un largo período en que los sueños mostraban, casi sin disfraz, su posición fálica; hasta que algo diferente empezó a emerger y el analista tomó partido por ese cambio. "Comenzó a festejarme un nuevo tipo de sueños. «Este es un sueño de castración, jla felicito!», dijo en varias ocasiones" (Rabinovich, 2015, p. 91). Aquí la intervención es nombrada, de manera muy original, como un "festejar" ciertos sueños. De esta manera, el proceder del analista intenta conducir la cura hacia lo que aparece como más promisorio en cuanto a la castración.

Algo similar relata otro pasante (AMP):

La relación con mi hijo adolescente todavía me daba esperanzas de ser algo. Un sueño: Estoy manejando una bicicleta que tiene un asiento para acompañante adelante. Yo manejo, él se divierte en el asiento. De repente, noto que él hace un recorrido propio con la bicicleta que yo no puedo controlar, pero hago de cuenta que lo controlo, acompañando sus movimientos. Él sigue su camino, ahora son dos bicicletas, yo no logro acompañarlo más, él es más rápido, ágil y está muy feliz por eso. Disminuyo la velocidad, él se aleja y admito que debo dejarlo seguir adelante. Él puede, y debe, seguir sin mí. "C'est comme ça?!" , escucho del analista. Nada de astucia u orgullo, apenas el vacío. Aún para mi hijo que depende directamente de mí, puedo faltar. Llegué a la nada. (da Silva, 2013, pp. 99-100)

Vemos que el analista remarca -incluso se podría decir aquí también que "festeja" - algo que el sueño muestra por sí mismo. El soñante se desprende de la necesidad de controlar los movimientos del otro, y tal vez se empieza a desprender del analista, cuya guía, en breve, ya no necesitará. El analista toma partido, remarca ese cambio expresado en la escena onírica.

Menos efusiva, la intervención de otro analista apunta también a destacar algo que el sueño muestra (AMP):

Un sueño de angustia en el que se proyectaba el movimiento del sujeto intentando ayudar a alguien de edad avanzada, de sexo indiscernible, recostado en una cama y que intenta incorporarse. Su boca abierta no puede pronunciar palabra. Al ir a ayudarle, el soñante es sacudido hacia atrás.

El analista solo le pedirá que escriba el sueño. En efecto, el sueño era una contracción del fantasma sobre un borde pulsional y se tratará de leer en esa página el goce que esas letras contenían. En el pase, el desciframiento del sueño le llevará a esclarecer el axioma del fantasma. (Palomera, 2006, párr. 35)

Aquí el analista simplemente interviene para pedir al analizante que ponga por escrito su sueño. Indicación desaconsejada por Freud (1932/2000), pero a menudo utilizada en el movimiento lacaniano para decantar algo que tiene que ver con el juego de las letras.

7En francés, "jasí es!".

Actualidades en Psicología, 34(129), 2020, 91-107 
"Un sueño es algo que no introduce a ninguna experiencia insondable, a ninguna mística: se lee en lo que se dice de él, y se podrá avanzar si se toman sus equívocos en el sentido más anagramático de la palabra", sostenía Lacan (1972-1973/2008, p. 116).

Podría decirse que, en última instancia, estas variantes del acto analítico frente al sueño, mostradas por los testimonios, coinciden en que subrayan lo que este muestra, especialmente cuando revela por sí mismo la castración o plantea cuestiones importantes para la cura.

\section{Discusión}

La hipótesis de inicio ha sido que una revisión de las intervenciones plasmadas en los testimonios de pase iba a mostrar que el trabajo con los sueños, en sus diversas modalidades, sigue vigente.

Ahora se puede decir que este análisis de los testimonios, seleccionados con el objetivo de visibilizar las intervenciones del analista, permite despejar el malentendido de creer que los sueños conclusivos se producirían sin el acto analítico, ya que, en verdad, este acompaña todo el tratamiento.

Las dos orientaciones de intervención sobre los sueños que se citaron en la introducción, a propósito de un artículo de López (2009), están presentes claramente en los ejemplos. Se tiene, por un lado, las intervenciones que apuntan a lograr un mayor despliegue asociativo, como lo son aquellas que interrogan elementos del sueño o invitan a asociar. $Y$, por el otro lado, aquellas intervenciones que intentan producir efectos interpretativos del estilo del corte. Se cree que es lícito incluir en este segundo grupo a la interpretación que juega sobre el significante y al corte de sesión, ya que ambas apuntan al más allá del sentido, pues apuntan a la letra.

Por otra parte, esta investigación ilumina lo que se ha llamado "variantes del acto analítico", donde se incluyen intervenciones que son interesantes por su modalidad descontracturada y original, verdaderamente dignas de lo que Lacan proclamó como "libertad táctica", cuando dijo que el analista debía ser libre de elegir el momento, el número y el estilo de sus intervenciones.

Es verdad, entonces, que, como sostiene Basz (2012), hay modos de intervenir sobre los sueños que no son equivalentes a la clásica interpretación de sentidos, ni se reducen al pedido de asociaciones, sino que, a través de estas otras modalidades -el corte, la interpretación que apunta a la letra y las variantes del acto analítico- se dirigen a un descompletamiento de los sentidos y a la caída progresiva de las identificaciones con los diversos elementos de la novela familiar.

\section{Referencias}

Basz, S. (2012). Respecto de los sueños, hay causa. En L. Ávola, A. Cucagna, \& A. Yacoi (Eds.), Brisas clínicas: sueño y final de análisis (pp. 97-103). Grama.

Brousse, M.H. (1997). Algunas observaciones sobre la interpretación a partir del Cartel del Pase. En N. Alvarez, P. Casalins, L. Michanie, A. Rubistein., \& F. Vitale (Eds.), Enseñanzas del pase (pp. 21-39). Publikar.

Dahan, P. (2010). Sobre lo vivo. Wunsch, 9, 27-29. 
da Silva, R. (2013). Pase Buenos Aires 2. Revista Lacaniana de Psicoanálisis, 14, 96-102.

Faig, C. (2012, Setiembre 17). El sueño de Aristóteles. El psicoanalista lector. http:// elpsicoanalistalector.blogspot.com.ar/2012/09/jacques-lacan-el-sueno-dearistoteles.html

Fajnwaks, F. (2016). Una mirada tan triste. Revista Lacaniana de Psicoanálisis, 20, 74-82.

Farías, F. (2011). Sueños del analizante, sueños del pasante. Wunsch, 10, 14-19.

Freud, S. (1900/2000). La interpretación de los sueños (J. L. Etcheverry, Trad.). En Obras Completas (Vols. 4-5). Amorrortu editores.

Freud, S. (1905/2000) El chiste y su relación con lo inconciente. En Obras Completas (J. L. Etcheverry, trad., Vol. 8). Amorrortu editores.

Freud, S. (1911/2001). El uso de la interpretación de los sueños en el psicoanálisis. (J. L. Etcheverry, Trad.). En Obras Completas (Vol. 12). Amorrortu editores.

Freud, S. (1912/2001). Consejos al médico sobre el tratamiento psicoanalítico (J. L. Etcheverry, Trad.). En Obras Completas (Vol. 12). Amorrortu editores.

Freud, S. (1919/1999). "Pegan a un niño". Contribución al conocimiento de la génesis de las perversiones sexuales (J. L. Etcheverry, Trad.). En Obras Completas (Vol. 17, pp. 173-200). Amorrortu editores.

Freud, S. (1923/2000). Observaciones sobre la teoría y práctica de la interpretación de los sueños (J. L. Etcheverry, Trad.). En Obras Completas (Vol. 19, pp. 107-122). Amorrortu editores.

Freud, S. (1932/2000). Nuevas conferencias de introducción al psicoanálisis. 29a conferencia: Revisión de la doctrina de los sueños. En Obras Completas (J. L. Etcheverry, trad., Vol. 22, pp. 7-28). Amorrortu editores.

Gasbarro, C. (2014). Testimonio I. Revista Lacaniana de Psicoanálisis, 17, 91-99.

Horne, B. (2012). Sobre el inicio del análisis por un sueño y su relación con el final. En L. Ávola, A. Cucagna., \& A. Yacoi, Brisas clínicas: sueño y final de análisis (pp. 35-42). Grama.

Klotz, J.P. (2000). Rêve et désir. L'essai. Revue Clinique Annuelle, 105-110.

Koretzky, C. (2012). Le réveil. Une élucidation psychanalytique. Presses Universitaires de Rennes.

Labaronnie, M. C. (2015) Notas sobre la subversión lacaniana del sueño. En Memorias del VII Congreso Internacional de Investigación y Práctica Profesional en Psicología XXII Jornadas de Investigación XI Encuentro de Investigadores en Psicología del MERCOSUR. Facultad de Psicología, Universidad de Buenos Aires. https://www. aacademica.org/000-015/781

Lacan, J. (1957/2003). La instancia de la letra en el inconsciente o la razón desde Freud. En Escritos 7 (pp. 473-509). Siglo XXI.

Lacan, J. (1958/2005). La dirección de la cura y los principios de su poder. En Escritos 2 (pp. 600-622). Siglo XXI.

Lacan, J. (1958-1959/2015). El deseo y su interpretación. En El seminario (Libro 6). Paidós. 
Lacan, J. (1967/2012). Proposición del 9 de octubre de 1967. En Otros Escritos (pp. 261277). Paidós.

Lacan, J. (1969/2012). El acto psicoanalítico. En Otros Escritos (pp. 395-403). Paidós.

Lacan, J. (1971/2012). Lituratierra. En Otros Escritos (pp. 19-29). Paidós.

Lacan, J. (1972-73/2008) Aun. En El seminario (libro 20). Paidós.

Lacan, J. (1973). Sobre la experiencia del pase. Ornicar?, 1, 31-44.

Lacan, J. (1973-1974). Los no incautos yerran (Los nombres del padre) (M.I. Agoff Trad.). En El seminario (libro 21) [Manuscrito inédito].

Lacan, J. (1975-1976/2009). El sinthome. En El seminario (libro 23). Paidós.

Lacan, J. (1976-1977). Lo no sabido que sabe de la una-equivocación se ampara en la morra. En El seminario (libro 24) [Manuscrito inédito]. Escuela Freudiana de Buenos Aires.

Lombardi, G. (1998). La resistencia como máscara del deseo. JVE Psiqué

López, H. (2009). Retorno a la interpretación de los sueños. Revista Universitaria de Psicoanálisis, 9, 126-146.

Lutterbach, A. L. (2009). Relato. En A. L. Lutterbach, E. Solano., \& F. Dassen (Eds.), Feminidad y fin de análisis (pp. 15-26). Grama.

Mazzuca, M. (2011). Ecos del pase. Letra Viva.

Miller, J.- A. (2007). La passe bis. Nouvelle revue de psychanalyse, 66, 209-213.

Miller, J.-A. (1973). Introducción a las paradojas del pase. Ornicar?, 1, 45-55.

Naparstek, F. (2001). La sesión analítica estructurada como el sueño. El caldero de la Escuela, 83, 146-150.

Naparstek, F. (2005). De la espera angustiosa a la serenidad del síntoma o Variaciones sobre la angustia y la espera. Revista Lacaniana de Psicoanálisis, 3, 51-55.

Nemirovsky, F. (2004). Un soñar sin angustia. Consecuancias clínicas. En F. Leguil, L. Noveau y F. Nemirovsky, Pase y transmisión 7 (pp. 23-26). Grama.

Nepomiachi, R. (1999). Sueños de pase. En S. Baudini, A. Luka, M. Recalde, P. Russo y L. Vignola (Eds.), Pase y transmisión 2 (pp. 31-36). E.O.L.

Palomera, V. (2006). Lo que el fantasma desconoce. Ornicar? Digital. http://wapol.org/ ornicar/articles/plm0127.htm

Rabinovich, D. (2015). Primer testimonio. El laberinto de mi deseo de saber. Revista Lacaniana de Psicoanálisis, 18, 89-97.

Rojas, R. (2017). Trazado de goce, interpretación y final. Wunsch, 16, 68-71.

Soler, C. (1988). Finales de análisis. Ediciones Manantial.

Straus, A., \& Corbin, J. (2016). Bases de la investigación cualitativa: técnicas y procedimientos para desarrollar la teoría fundamentada. Universidad de Antioquia.

Tarrab, M. (25 de abril, 2006). Y el soplo se vuelve signo. Escuela de la Orientación Lacaniana. http://www.eol.org.ar/template.asp? Sec=el pase\&SubSec=testimoni os\&File=testimonios/tarrab soplo.html

Actualidades en Psicología, 34(129), 2020, 91-107 
Thamer, E. (2018). Un psicoanálisis no puede todo. Wunsch, 17, 5-7.

Vidal, C. (2015). Niebla. Testimonio de pase Vigo 1 de octubre de 2015. [Inédito].

Vinciguerra, R.-P. (2006). Había... hay. Escuela de la Orientación Lacaniana. http://www. eol.org.ar/template.asp? Sec=el paseySubSec=testimoniosyFile=testimonios/ vinciguerra habia.html

Yacoi, A. (2002). Sueños en la conclusión de los análisis. Mediodichos.

Yacoi, A. (2012). Sueño y fin de análisis, una introducción. En L. Ávola, A. Cucagna, y A. Yacoi, Brisas clínicas: sueño y final de análisis (pp. 129-133). Grama. 